Check for updates

Cite this: RSC Adv., 2019, 9, 2756

Received 6th October 2018

Accepted 5th January 2019

DOI: $10.1039 / \mathrm{c} 8 \mathrm{ra0} 8272 \mathrm{c}$

rsc.li/rsc-advances

\section{A UPLC-MS/MS application for comparisons of the hepatotoxicity of raw and processed Xanthii Fructus by energy metabolites $\uparrow$}

\author{
Hai Jiang,,$^{a}$ Liu Yang, (D) $\ddagger^{a}$ Xudong Xing, (DD ${ }^{a}$ Meiling Yan, ${ }^{a}$ Xinyue Guo, ${ }^{a}$ Ajiao Hou, ${ }^{a}$ \\ Wenjing Man, ${ }^{a}$ Bingyou Yang, ${ }^{a}$ Qiuhong Wang ${ }^{\star a b}$ and Haixue Kuang ${ }^{\star a}$
}

\begin{abstract}
The ripe fruit of Xanthium strumarium L. (Xanthii Fructus) cannot be widely used as a Chinese herbal medicine (CHM) owing to its hepatotoxicity. However, Xanthii Fructus (XF) can be used effectively and safely after correct processing based on traditional experience, although a high hepatotoxicity risk remains owing to improper usage. Therefore, the processing methods used must be clarified to ensure safety. The adenosine$5^{\prime}$-triphosphate (ATP) level in tissues is an important indicator reflecting the functional status of liver cells. Therefore, this study aims to evaluate the hepatotoxicity of XF using UPLC-MS/MS. The hepatotoxicity of raw XF (RXF) and XF processed by intermediary energy metabolites (PXF) is compared. The method is evaluated for its analytical performance and successfully applied to the quantification of ATP, adenosine- $5^{\prime}$-diphosphate (ADP), adenosine-5'-monophosphate (AMP), atractyloside, and carboxyatractyloside in mouse liver. The hepatotoxicity results also indicate that the toxicity of XF is decreased after processing, perhaps due to the decrease in atractyloside and carboxyatractyloside contents. Importantly, the experimental evidence provides a rationale for the reduction in toxicity. These data show that mouse livers are damaged between the days 20 and 30 of RXF oral administration, and that the ATP level is decreased. Importantly, no significant difference is observed between the PXF treatment group and control group, while the RXF treatment group is significantly different. Therefore, processing can reduce the toxicity of XF.
\end{abstract}

\section{Introduction}

In the liver, drugs undergo enzymatic biotransformations that enhance metabolite hydrophilicity and clearance from the body. The liver is the most frequently damaged organ, and its failure can result in death. ${ }^{1,2}$ Importantly, medication-induced hepatotoxicity is the single most important reason for both the rejection of drugs and withdrawal of drugs from the market after approval by the United States Food and Drug Administration. Reducing hepatotoxicity during drug development might be possible using innovative preclinical hepatotoxicity screening methods. ${ }^{3,4}$ The World Health Organization estimates that $65-80 \%$ of the world's population uses primary forms of herbs in healthcare. ${ }^{5}$ Natural medicine therapy in China has a long history of more than 2000 years. Although Chinese herbal medicines (CHMs) have been used to treat chronic and

${ }^{a}$ Key Laboratory of Chinese Materia Medica, Heilongjiang University of Chinese Medicine, Ministry of Education, Harbin, 150040, PR China. E-mail: hxkuang56@ 163.com; qhwang668@sina.com

${ }^{b}$ School of Traditional Chinese Medicine, Guangdong Pharmaceutical University, Guangzhou, 528458, PR China

$\dagger$ Electronic supplementary information (ESI) available. See DOI: 10.1039/c8ra08272c

\$ These authors equally contributed to this work. intractable diseases with a definite therapeutic effect, they are now confronted with the major challenge of potential gradual hepatotoxicity. This seriously affects the quality of CHMs and is of great importance in present research and development into safe and effective CHMs for disease treatment.

Xanthii Fructus (XF) is the ripe fruit of Xanthium strumarium L., which belongs to the Asteraceae family. XF is widely distributed in Asia, Europe, and America. ${ }^{6}$ In recent years, $\mathrm{XF}$ has attracted increasing attention in the pharmaceuticals industry owing to its therapeutic benefits, such as in the treatment of nasal sinusitis, and headache caused by rheumatism and skin pruritus. ${ }^{7,8} \mathrm{XF}$ also possesses numerous significant pharmacological properties, such as anti-inflammatory, ${ }^{9}$ antiproliferative, ${ }^{10}$ antioxidant, ${ }^{11}$ antiarthritic, ${ }^{12}$ analgesic ${ }^{7}$ and antiallergic rhinitis activities. ${ }^{13}$ The chemical components in XF include essential oils, phenolic acids, lignin, glycosides, and monoterpene glucosides. ${ }^{14-16}$ In recent decades, increasing toxicity incidents have occurred through improper use of XF. ${ }^{17-19}$

In traditional Chinese medicine, processing is an important procedure believed to decrease the toxicity and alter the therapeutic efficacy of CHMs. ${ }^{20,21}$ Accordingly, CHM processing plays an important role in ensuring the safe use of potentially toxic herbs. To characterize the chemical compositions of XF or processed XF (PXF), many analytical methods have been developed. ${ }^{22,23}$ Various assays have been established to 
determine raw material hepatotoxicity. However, hepatotoxicity determination of XF and PXF using a simple chromatographic method has yet to be reported.

The liver is an important organ for energy metabolism and conversion in the body. Concomitantly, hepatocytes are rich in active mitochondria, providing enough energy for cellular metabolism. Liver damage impairs the energy metabolism of hepatocyte mitochondria, ${ }^{\mathbf{2 4}}$ implying alterations in energy biomolecules. Therefore, the simultaneous quantification of adenosine- $5^{\prime}$-triphosphate (ATP), adenosine- $5^{\prime}$-diphosphate (ADP), and adenosine- $5^{\prime}$-monophosphate (AMP) in liver tissue is important for understanding the energy state and evaluating the hepatotoxicity of XF. Various methods, such as NMR spectroscopy, ${ }^{25}$ capillary electrophoresis, ${ }^{26}$ and aptamer-based optical sensing, ${ }^{27,28}$ have been developed for ATP, ADP, and AMP analysis. However, most of these methods suffer from poor selectivity and multistep reactions of complex compounds. Furthermore, some of these methods produce large background signals. Ultra-performance liquid chromatography with tandem mass spectrometry (UPLC-MS/MS) is a preferable method owing to its high sensitivity and selectivity, and has been widely used in plant and biological analysis. Another advantage of UPLCMS/MS is the simultaneous measurement of multiple analytes in a single run with a short separation time. Accordingly, considerable efforts have been devoted toward developing UPLC-MS/MS for ATP, ADP, and AMP analysis.

A fast, simple, and comprehensive method is urgently needed to assess the hepatotoxicity of XF and PXF. Therefore, in this study, UPLC-MS/MS was used to simultaneous determine ATP, ADP, and AMP levels in the livers of mice orally administered with raw XF (RXF) and PXF. In previous reports, diterpenoid glycoside atractyloside (ATR) and its derivative carboxyatractyloside (CATR) were the principal toxic compounds found in XF. ${ }^{29,30}$ This UPLC-MS/MS method has been evaluated and applied to the quantitative determination of ATR and CATR in mouse liver for the first time. The results will clarify the hepatotoxicity caused by cumulative amounts of ATR and CATR in mouse liver after different periods of oral administration of RXF and PXF. This method could be applied in many fields, from drug discovery to cell biology, and further customized to include other related energy metabolites in future.

\section{Materials and methods}

\section{Chemicals and reagents}

ATP, ADP, and AMP standards were purchased from Sigma (St. Louis, MO, USA). Internal standards (chloramphenicol) were also obtained from Sigma (St. Louis, MO, USA). ATR and CATR were purchased from Chengdu Must Bio-technology Co., Ltd. (Chengdu, China) with purities of $>99.0 \%$. The chemical structures of the five analytes are shown in Fig. S1. $\dagger$ HPLC-grade methanol and acetonitrile were supplied by Fisher Scientific (Pittsburgh, PA, USA). Formic acid and ammonium acetate were purchased from Dikma Co. (USA), and water used was Wahaha purified water purchased from the Hangzhou Wahaha group (Hangzhou, China). Other reagents and chemicals were all of analytical grade.

\section{Animal experiments}

A total of 54 male adult mice aged 3-4 months were used as subjects and kept in an air-conditioned room at a constant room temperature of $24 \pm 2{ }^{\circ} \mathrm{C}$, relative humidity level of $50 \pm$ $15 \%$, and a $12 \mathrm{~h} \mathrm{light/dark} \mathrm{cycle} \mathrm{with} \mathrm{free} \mathrm{access} \mathrm{to} \mathrm{food} \mathrm{and}$ water. The animals were acclimatized for a period of one week. This experiment was approved by the Animal Ethics Committee of Heilongjiang University of Chinese Medicine. According to the Chinese pharmacopeia (2015 edition), the routine dose of $\mathrm{XF}$ for humans is $3-10 \mathrm{~g}$ per day. The mice were divided into three groups of 18 animals each as follows: Group I was administered orally with RXF extract at a dose of $120.0 \mathrm{~g} \mathrm{~kg}^{-1}$; group II was administered orally with PXF extract at the same dose; and group III was the normal control group administered with an equal volume of normal saline. After oral administration, the mice were sacrificed through bleeding of the femoral artery. The liver was promptly removed after sacrificing the animals on days 11,21 , and 31 of oral administration, using six animals randomly selected from each group, respectively.

\section{Sample preparation}

Freeze-dried $\left(-70{ }^{\circ} \mathrm{C}\right.$ for $\left.36 \mathrm{~h}\right)$ mouse liver samples were quickly ground and blended in a mortar. Powdered liver tissue (10 mg) was then placed into a $1.5 \mathrm{~mL}$ scaled Eppendorf tube containing pre-cooled $\left(4{ }^{\circ} \mathrm{C}\right)$ methanol $(500 \mu \mathrm{L})$ and proteins were precipitated by vortex shaking for $1 \mathrm{~min}$. Pre-cooled $\left(4^{\circ} \mathrm{C}\right)$ water $(498$ $\mu \mathrm{L})$ and IS $(2 \mu \mathrm{L})$ were then immediately added to extract the analytes by vortex shaking for $3 \mathrm{~min}$. After centrifugation of the extract at $16000 \times g$ and $-10{ }^{\circ} \mathrm{C}$ for $20 \mathrm{~min}$, the supernatant was stored at $-20{ }^{\circ} \mathrm{C}$ prior to UPLC-MS/MS analysis.

\section{Instrumentation and UPLC-MS/MS conditions}

Ultra-high performance liquid chromatography (Thermo Scientific, Vanquish) was performed on a Hypersil GOLD column $(100 \mathrm{~mm} \times 2.1 \mathrm{~mm}, 1.9 \mu \mathrm{m})$ using a mobile phase consisting of (A) $2 \mathrm{mM}$ ammonium acetate in water and (B) acetonitrile. The optimized UPLC elution conditions were as follows: $0-2 \mathrm{~min}, 5 \% \mathrm{~B} ; 5-8 \mathrm{~min}, 5-30 \% \mathrm{~B}$. The column temperature was maintained at $45{ }^{\circ} \mathrm{C}$.

Mass spectrometry analysis was performed using a TSQ Quantis Triple Quadrupole Mass Spectrometer (Thermo Fisher Scientific, USA) with selected reaction monitoring (SRM), an online degasser, and a binary pump solvent management system. A negative-ion scan was used to obtain precursor ions and product ion spectra of ATP, ADP, AMP, diterpenoid glycoside, and IS. Optimized parameters for the five analytes and IS are listed in Table 1. Other optimized MS parameters were as follows: spray voltage, $3000 \mathrm{~V}$; ion transfer tube and vaporizer temperatures, $325{ }^{\circ} \mathrm{C}$ and $350{ }^{\circ} \mathrm{C}$, respectively; Aux gas flow rate, $2 \mathrm{~L} \mathrm{~min}{ }^{-1}$.

\section{Preparation of stock standard solutions and quality control samples}

Stock solutions of the analytes were mixed and diluted to afford a final mixed standard solution containing ATP $\left(1.175 \mathrm{mg} \mathrm{mL}^{-1}\right)$, ADP (1.136 mg mL ${ }^{-1}$ ), AMP (1.414 mg mL $\left.{ }^{-1}\right)$, ATR (1.170 mg 
Table 1 Optimized SRM parameters of five analytes and IS

\begin{tabular}{|c|c|c|c|c|c|c|}
\hline Peak no. & Analytes & $\begin{array}{l}\text { Retention time } \\
(\mathrm{min})\end{array}$ & $\begin{array}{l}\text { Precursor ion } \\
\left([\mathbf{M}-\mathbf{H}]^{-}\right)(m / z)\end{array}$ & $\begin{array}{l}\text { Product ion } \\
(\mathrm{m} / \mathrm{z})\end{array}$ & $\begin{array}{l}\text { Collision energy } \\
\text { (V) }\end{array}$ & $\begin{array}{l}\text { Dwell time } \\
\text { (s) }\end{array}$ \\
\hline 1 & ATP & 0.97 & 506 & 273 & 26.83 & 0.8 \\
\hline \multirow[t]{2}{*}{2} & $\mathrm{ADP}$ & 0.98 & 426 & 328 & 16.56 & 0.8 \\
\hline & & & & 408 & 18.98 & \\
\hline 3 & AMP & 0.99 & 346 & 134 & 31.34 & 0.8 \\
\hline & & & & 645 & 32.23 & \\
\hline \multirow[t]{2}{*}{5} & ATR & 4.90 & 725.0 & 543 & 48.33 & 0.8 \\
\hline & & & & 645 & 23.45 & \\
\hline \multirow[t]{2}{*}{6} & Chloramphenicol (IS) & 6.22 & 321.0 & 152 & 10.23 & 0.8 \\
\hline & & & & 257 & 10.23 & \\
\hline
\end{tabular}

$\left.\mathrm{mL}^{-1}\right)$, and CATR (1.450 $\mathrm{mg} \mathrm{mL}^{-1}$ ). A mixed series of working solutions of these five compounds were prepared by further diluting the mixed standard solution in appropriate ratios. Chloramphenicol (IS) was prepared and diluted to $100 \mathrm{ng} \mathrm{\textrm {mL } ^ { - 1 }}$ using methanol. Quality control samples at high, medium, and low concentrations were obtained in the same manner. All samples were stored at $-20{ }^{\circ} \mathrm{C}$ and working solutions were allowed to warm to room temperature before injection.

\section{Preparation of RXF and PXF extracts}

RXF was extracted by reflux with $50 \%$ ethanol three times. The ethanol extracts were combined and evaporated to dryness under reduced pressure with a vacuum rotary evaporator. The extract was suspended in water with $0.1 \% \mathrm{w} / \mathrm{v}$ sodium carboxyl methylcellulose for oral administration. PXF was prepared using a procedure previously reported by our research group. ${ }^{31}$ The same procedure was adopted to prepare the PXF extract, which was stored at $4{ }^{\circ} \mathrm{C}$. The ATR and CATR contents in the RXF and PXF extracts were determined, as shown in Table S1. $\dagger$

\section{Method validation}

The current UPCL-MS/MS assay was validated for selectivity, linearity, precision, accuracy, extraction recovery, and stability, in accordance with the requirements for biological sample analysis.

Selectivity and specificity. The selectivity of the approach was evaluated by analysing blank liver homogenate samples. The methanol-water $(1: 1, \mathrm{v} / \mathrm{v})$ solution, RXF extract, blank tissue homogenate sample with IS, and real tissue homogenate samples collected from treated rats were compared by chromatography. All-blank tissue homogenate samples were prepared and analysed to ensure the absence of interfering peaks.

Linearity and sensitivity. Using $1 / x^{2}$ as the weighting factor, linear regression equations of the five analytes were obtained by plotting the analyte-to-IS peak area ratio $(y) v s$. the analyte concentration $(x)$. The lower limit of quantification (LLOQ) was determined in accordance with the base line noise at a signal-tonoise ratio of $10: 1$. The target constituent concentrations showed satisfactory accuracy errors $( \pm 20 \%)$ and precision variation $(<20 \%)$.

Precision and accuracy. The precision of this established method was confirmed through CATR and ATR determination in six batches of quality control samples at three different concentrations (low, medium, and high). To evaluate the intraday and inter-day precisions, the three-level quality control samples were tested for one day and three consecutive days, respectively. The accuracy of this approach toward ATP, ADP, and AMP determination was evaluated by recovery test using the standard addition method. Spiked sample solutions were prepared at three different concentrations using the previously described method and then triplicate experiments were performed at each level. The recovery percentages were calculated using the equation: recovery $(\%)=[$ (total detected amount original amount)/added amount $] \times 100$. The calibration curves above were then used to determine the quantities of the five analytes in the tested samples. Acceptable criteria for the analysis were a precision of less than 15\% and an accuracy within $\pm 15 \%$.

Extraction recovery and matrix effect. Extraction recoveries of ATR and CATR were investigated using three-level quality control samples by calculating the ratio of mean peak area of the analytes in the extracted livers samples to the area of postextracted samples spiked with the target constituents at the same concentration level. The matrix effect at different quality control levels was measured by comparing the peak area ratio of prepared blank livers samples with analytes added to those dissolved in the initial mobile phase at equal concentrations in six replicates.

Stability. To test the stability of the five analytes, the quality control samples were measured at day 0 , then stored at $-20{ }^{\circ} \mathrm{C}$ for 7 days and remeasured. To test the autosampler stability, the analytes at three concentration levels in the quality control samples were assayed at $4{ }^{\circ} \mathrm{C}$ for $24 \mathrm{~h}$.

\section{Statistical analysis}

The experimental data were expressed as means \pm standards deviation (SD, $n=6$ ). One-way ANOVA using Tukey's multiple comparison test was performed to determine differences between 
groups. Results were considered significant when $p<0.05$. Calculations were performed using GraphPad Prism 5.00 vision.

\section{Results and discussion}

\section{Optimization of UPLC-MS/MS conditions}

The composition of the mobile phase was optimized through several steps on the Hypersil GOLD column. Firstly, the effect of methanol and acetonitrile as organic mobile phases using the same LC gradient was investigated. The analytes were not eluted after 20 min when acetonitrile was substituted with methanol. This showed that acetonitrile was much more effective at displacing adenine nucleotides from the Hypersil GOLD column, exhibiting a stronger elution capability than methanol. Therefore, acetonitrile was selected for further experiments in this study. Secondly, a solvent system containing $0.1 \%$ formic acid and different concentrations of ammonium acetate $(2,5$, and $10 \mathrm{mM})$ were found to be optimal for separation of these analytes. When $0.1 \%$ formic acid was added to the mobile phase, no good peak pattern was observed for ATR and CATR. In contrast, when ammonium acetate was added to the mobile phase, five analytes showed good peak shapes. However, increasing the salt concentration suppressed the MS signal intensity owing to salt precipitation in the ion source and ion transportation tube. Therefore, $2 \mathrm{mM}$ ammonium acetate was added to the mobile phase, with reasonable retention and separation, and good sensitivity and reproducibility, obtained.

SRM mode was used to detect the complicated target constituents to improvement the selectivity and sensitivity. In precursor ion full scan mode, deprotonated molecules $[\mathrm{M}-\mathrm{H}]^{-}$were detected with abundant ions for analysis, while protonated molecules $[\mathrm{M}+\mathrm{H}]^{+}$were observed with more abundant intensities. Other parameters, including SRM transitions, collision energy, and cone voltage, were also optimized to achieve the highest intensities of deprotonated or protonated molecules of analytes using the syringe pump program. The MS spectra of ATP, ADP, AMP, ATR, and CATR showed deprotonated precursor ions [M $\mathrm{H}]^{-}$at $m / z 506,426,346,725$, and 792, respectively in the Q1 scan. The $[\mathrm{M}-\mathrm{H}]^{-}$ions of ATP, ADP, AMP, ATR, and CATR yielded abundant product ions at $m / z 355,224,225,188$, and 280 in the Q3, which were selected as SRM transitions for quantitation (Table 1). Fig. 1 shows the UPLC-MS/MS chromatogram of a standard mixture of the five target compounds in SRM mode.

\section{Method validation}

Selectivity and specificity. Representative SRM chromatograms were obtained for a blank liver sample (Fig. S2A $\dagger$ ), methanol-water $(1: 1, \mathrm{v} / \mathrm{v})$ solution (Fig. S2B $\dagger$ ), blank liver sample spiked with three analytes and IS (Fig. 1A), and RXF extract with IS (Fig. 1B). The retention times of ATP, ADP, AMP, ATR, CATR, and IS were approximately 0.97, 0.98, 0.99, 3.85, 4.90 , and $6.22 \mathrm{~min}$, respectively. No significant interferences from endogenous ingredients were observed in the UPLC-MS/ MS analysis of the five constituents and IS.

Linearity and LLOQ. The standard curves of the five analytes all exhibited good linearity with correlation coefficients $\left(R^{2}\right)$ of $>0.999$. The linear ranges, regression equations, LLOQs, and coefficients obtained from typical calibration curves are shown in Table 2. The LLOQs were appropriate for quantitative detection of the five analytes in the studies.

Precision and accuracy. The accuracy of ATP, ADP, and AMP determination was evaluated using the recoveries of spiked liver tissue samples, while precision was evaluated using the relative standard deviations (RSDs). Three concentration levels of the mixture standard solutions were added to each sample and then analysed using the established method, with six replicates performed for each level. The results are shown in Table S2. $\dagger$ For the three spiked levels, the average recoveries ranged from $97.7 \%$ to $101.4 \%$. The RSDs of the method ranged from $0.26 \%$ to $2.43 \%$. For CATR and ATR, the intra- and inter-day precision and accuracy were determined by measuring quality control samples at three concentration levels. The accuracy was expressed by the relative error (RE). The precision and accuracy for CATR and ATR are shown in Table $\mathrm{S} 3 . \dagger$ The intra- and inter-day precisions were 1.58-8.23\% and $2.02-9.08 \%$, respectively. The accuracy derived from quality control samples was between $-6.21 \%$ and $8.27 \%$ at the three quality control levels. All results indicated that the overall reproducibility of the method was acceptable.

Extraction recovery and matrix effect. Table $\mathrm{S} 4 \uparrow$ summarizes the extraction recoveries and matrix effects of the two analytes and IS in the quality control samples. The mean extraction recoveries of the investigated components in liver samples at three different concentration levels were found to be 86.35$97.57 \%$ with RSDs of $<6.86 \%$, which indicated that the recovery of this method was precise and appropriate at the different levels. The matrix effects ranged from 86.49 to $100.5 \%$ at the three quality control levels. Therefore, no obvious matrix effects were observed in the target compound and IS analyses in this investigation, showing that endogenous ingredients did not interfere with the ionization of analytes and IS.

Stability. Stability evaluation showed a slight decrease in the signal intensity after storing at $-20{ }^{\circ} \mathrm{C}$ for one week. This slight decrease showed that samples (Table $55 \dagger$ ) can be stored at $-20{ }^{\circ} \mathrm{C}$ for a short period of time prior to analysis without having a major adverse effects on the results.

\section{Comparing ATP, ADP, and AMP levels in liver}

This method was successfully applied to the determination of ATP, ADP, and AMP levels in liver tissue after 10, 20, and 30 days of oral administration. The results are summarized in Fig. 2. No significant change in ATP, ADP, and AMP levels was observed after 10 days of oral administration of normal saline, RXF, and PXF $(p>0.05)$ (Fig. 2A). The ATP levels in the RXF group had decreased after 20 days of oral administration. However, there was no significant difference compared with the control group $(p>0.05)$ (Fig. 2B). RXF administration markedly decreased the ATP content $(p<0.01)$, while the ADP levels had increased (Fig. 2C). The ATP, ADP, and AMP levels are closely related to liver function. Therefore, the mouse livers analysed on the 20th and 30th days of oral administration of RXF were damaged, resulting in decreased ATP levels. Importantly, the PXF and control groups showed no significant differences, while the RXF group was significantly different. 
Overall, the above animal experiments suggested that processing significantly decreased the toxicity of RXF.

\section{Quantitative analysis of ATR and CATR in liver}

Some studies have attributed hepatotoxicity to ATR and CATR contents. $^{32}$ ATR and CATR are both specific and powerful inhibitors of the mitochondrial ADP/ATP transport system, and interact with the ADP/ATP carrier protein, named the adenine nucleotide translocator (ANT), in the inner mitochondrial membrane. ${ }^{30,33}$ As shown in Fig. 3, as the number of days of RXF oral administration increased, the amount of CTR and CATR in the mouse liver tissue also increased. Compared
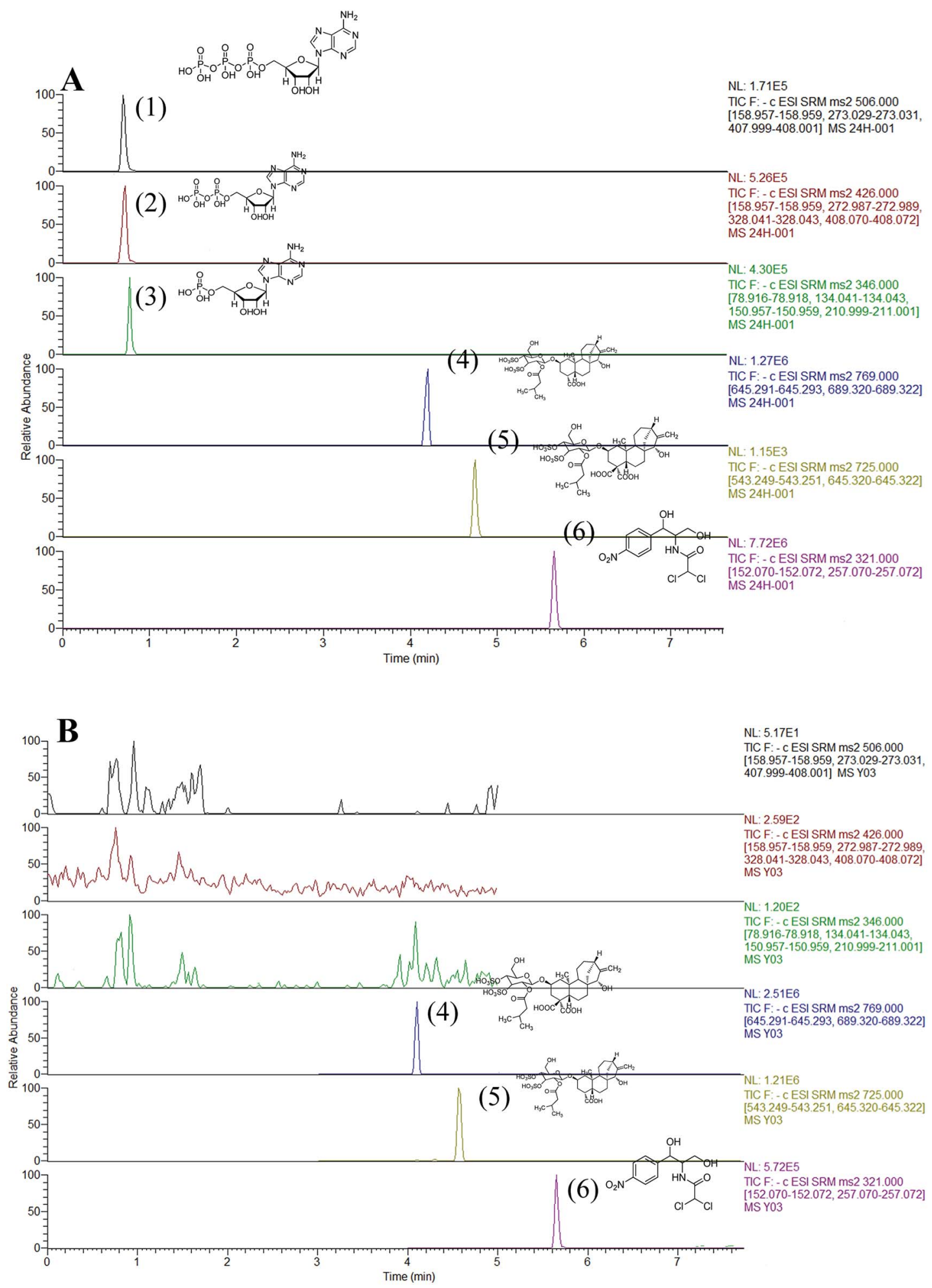

Fig. 1 UPLC-MS/MS analysis SRM chromatogram of five analytes: ATP (1), ADP (2), AMP (3), ATR (4), CATR (5), and IS (6) in different matrices. 
Table 2 Regression equation, correlation coefficients, linearity ranges, and LLOQs for five analytes

\begin{tabular}{|c|c|c|c|c|c|}
\hline Peak no. & Analytes & Calibration curves & $\begin{array}{l}\text { Correlation } \\
\text { coefficient, } R^{2}\end{array}$ & $\begin{array}{l}\text { Linear range } \\
\left(\mathrm{ng} \mathrm{mL}^{-1}\right)\end{array}$ & $\begin{array}{l}\text { LLOQs } \\
\left(\mathrm{ng} \mathrm{mL} \mathrm{mL}^{-1}\right)\end{array}$ \\
\hline 1 & ATP & $y=0.0130 x-0.8114$ & 0.9990 & $10.5-1080.5$ & 10.5 \\
\hline 2 & ADP & $y=0.0270 x-2.4892$ & 0.9991 & 9.5-1180.5 & 9.5 \\
\hline 3 & AMP & $y=0.0101 x-0.7401$ & 0.9990 & $10.6-2070.0$ & 10.6 \\
\hline 4 & CATR & $y=0.0006 x-0.0271$ & 0.9992 & $9.5-1450.5$ & 9.5 \\
\hline 5 & ATR & $y=0.0048 x-0.1623$ & 0.9996 & $10.8-1480.0$ & 10.8 \\
\hline
\end{tabular}
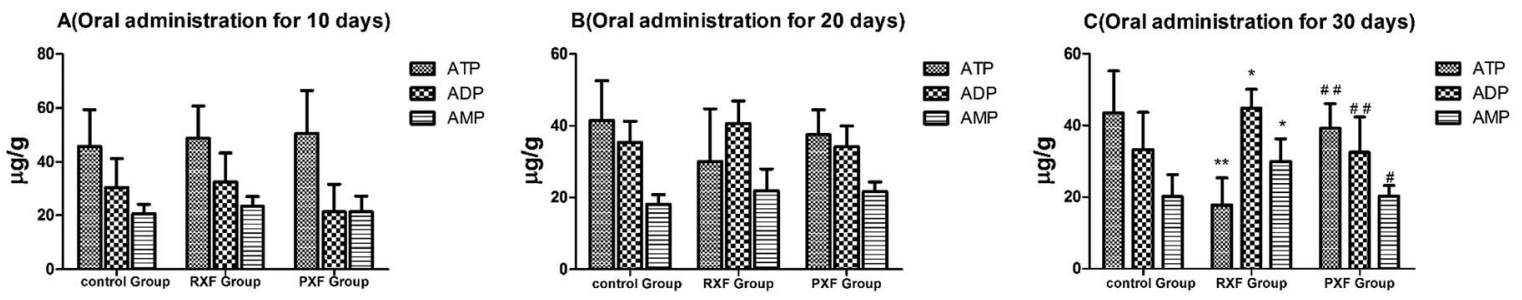

Fig. 2 ATP, ADP, and AMP levels in mice liver tissue 10, 20, and 30 days after oral administration. Values are means \pm SD from six animals in each group. ${ }^{*} p<0.05,{ }^{* *} p<0.01$ vs. control. ${ }^{*} p<0.05,{ }^{\# \#} p<0.01$ vs. RXF group.

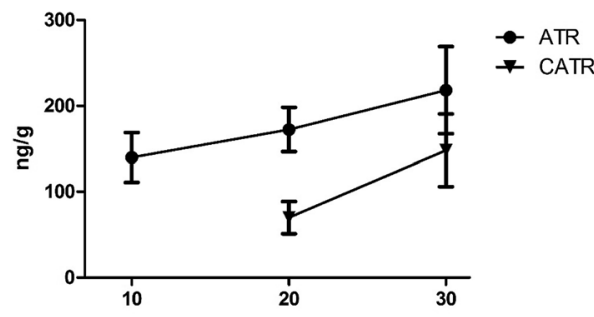

Fig. 3 ATR and CATR contents in liver tissue of ground RXF.

with the control group, the ATP levels in the RXF and control groups were significantly different after oral administration for 30 days. However, the CATR content was only detected in some of the 10 day oral treatment RXF group. This might be attributed to the CATR content in mouse liver being below the LLOQ, or CATR combining with the ADP/ATP carrier in mouse liver, because these compounds are diterpene glycosides with one isovaleric and two sulfate groups, while CATR possesses an extra carboxylate group, which confers a 10-fold increase in affinity. ${ }^{33,34}$ For the PXF group, ATR and CATR were not detected in all liver tissues after 10 and 20 days of oral administration. After 30 days of oral administration, ATR and CATR were detected in only some of the mouse livers (Tables S6 and S7 $\dagger$ ). Meanwhile, both the ATR and CATR levels were found to be reduced in our previous studies. ${ }^{31}$ This might be due to the ATR and CATR levels in PXF being lower than those in RXF. Furthermore, the CHM was composed of multiple components, which might be changed by processing, which would affect the tissue distribution of CATR and ATR. Further study is needed to determine the mechanisms through which processing affects the XF composition.

\section{Conclusions}

This UPLC-MS/MS method has been successfully applied to the determination of ATP, ADP, and AMP in liver tissue from mice orally administered for 10,20 , and 30 days. The levels of ATP, $\mathrm{ADP}$, and AMP were closely related to the liver function. Therefore, mouse livers were damaged by $20-30$ days of oral administration of RXF, which decreased the ATP levels. Importantly, there was no significant difference between the PXF and control groups, while the RXF group showed significant differences. These data showed that processing could reduce the toxicity of RXF.

In summary, processing significantly decreased the hepatotoxicity of RXF. Processing CHMs is common practice and usually occurs before herbs are prescribed. Therefore, processing is necessary for the application of CHMs in clinical use.

\section{Conflicts of interest}

There are no conflicts of interest to declare.

\section{Acknowledgements}

This work was supported financially by the National Natural Science Foundation of China (Grant No. 81703684 and 81803690), the Natural Science Foundation of Heilongjiang Province (No. H2015037), the Postdoctoral Initial Fund of Heilongjiang Province, the University Nursing Program for Young Scholars with Creative Talents in Heilongjiang Province (No. UNPYSCT-2017215), the Heilongjiang University of Chinese Medicine Doctoral Innovation Foundation (No. 2014bs05), the Application Technology Research and Development Projects of Harbin Technology Bureau (No. 2014RFQXJ149), the Innovative Talents Funding of Heilongjiang University of Chinese 
Medicine, the Heilongjiang (No. 2018RCD25) Postdoctoral Scientific Research Developmental Fund (No. LBH-Q16210), the Heilongjiang University of Chinese Medicine Doctoral Innovation Foundation (No. 2013bs04), the University Nursing Program for Young Scholars with Creative Talents in Heilongjiang Province (No. UNPYSCT-2017219), and Postgraduate Funds for Heilongjiang University of Chinese Medicine (2018YJSCX061).

\section{Notes and references}

1 M. Z. Sakatis, M. J. Reese, A. W. Harrell, M. A. Taylor, I. A. Baines, L. Chen and C. A. Lovatt, Chem. Res. Toxicol., 2012, 25, 2067-2208.

2 A. Srivastava, J. L. Maggs, D. J. Antoine, D. P. Williams, D. A. Smith and B. K. Park, Handb. Exp. Pharmacol., 2010, 165-194.

3 J. A. Dimasi, Clin. Pharmacol. Ther., 2001, 69, 297-307.

4 M. W. Tengowski and J. J. Kotyk, Risk identification and management: In Imaging in Drug Discovery and Early Clinical Trials, 2005, pp. 257-278.

5 Y. T. Zhang, M. F. Xiao, K. W. Deng, Y. T. Yang, Y. Q. Zhou, J. Zhou and W. L. Liu, Phytomedicine, 2018, 45, 68-75.

6 A. Kamboj and A. K. Saluja, Int. J. Green Pharm., 2010, 4(3), 129.

7 T. Han, H. L. Li, Q. Y. Zhang, P. Han, H. C. Zheng, K. Rahman and L. P. Qin, Phytomedicine, 2007, 14(12), 825-829.

8 M. H. Huang, B. S. Wang, C. S. Chiu, S. Amagaya, W. T. Hsieh, S. S. Huang and G. J. Huang, J. Ethnopharmacol., 2011, 135(2), 545-552.

9 H. Jiang, L. Yang, G. X. Ma, X. D. Xing, M. L. Yan, Y. Y. Zhang and X. D. Xu, Fitoterapia, 2017, 117, 11-15.

10 H. Jiang, G. X. Ma, L. Yang, X. D. Xing, M. L. Yan, Y. Y. Zhang and X. D. Xu, Phytochem. Lett., 2016, 18, 192-196.

11 A. S. Ingawale, M. B. Sadiq, L. T. Nguyen and T. B. Ngan, Biocatal. Agric. Biotechnol., 2018, 14, 40-47.

12 B. Lin, Y. Zhao, P. Han, W. Yue, X. Q. Ma, K. Rahman and T. Han, J. Ethnopharmacol., 2014, 155(1), 248-255.

13 W. Peng, Q. L. Ming, P. Han, et al., Phytomedicine, 2014, 21(6), 824-829.

14 G. Vial, H. Dubouchaud, K. Couturier, C. Cottet-Rousselle, N. Taleux, A. Athias and X. M. Leverve, J. Hepatol., 2011, 54(2), 348-356.

15 H. Jiang, X. Xing, M. Yan, X. Guo, L. Yang and L. Yang, Nat. Prod. Res., 2018, 1-6.
16 J. Hai, Y. Liu, X. D. Xing, Y. Y. Zhang, M. L. Yan and B. Y. Yang, Chin. Tradit. Herb. Drugs, 2017, 48(1), 47-51.

17 R. Teschke, L. Zhang, H. Long, A. Schwarzenboeck, W. Schmidt-Taenzer, A. Genthner and A. Wiebrecht, Deutsche Zeitschrift für Akupunktur, 2016, 59(4), 33-35.

18 H. Karabiber, H. Almis, M. A. Selimoglu, C. Yakinci and S. Yilmaz, J. Pediatr. Gastroenterol. Nutr., 2014, 58(1), e6-e9.

19 P. L. West, N. J. Mckeown and R. G. Hendrickson, Clin. Toxicol., 2008, 46(7), 609.

20 S. Liu, F. Li, Y. Li, W. Li, J. Xu and H. Du, J. Ethnopharmacol., 2017, 207, 237-250.

21 Z. Zhao, Z. Liang, K. Chan, G. Lu, E. L. M. Lee, H. Chen and L. Li, Planta Med., 2010, 76(17), 1975-1986.

22 H. Jiang, L. Yang, X. Xing, M. Yan, X. Guo, B. Yang and H. X. Kuang, J. Pharm. Biomed. Anal., 2018, 153, 117.

23 H. Jiang, L. Yang, X. Xing, M. Yan, X. Guo, B. Yang and H. Kuang, Molecules, 2018, 23(2), 243.

24 C. Koliaki, J. Szendroedi, K. Kaul, T. Jelenik, P. Nowotny, F. Jankowiak and M. Schlensak, Cell Metab., 2015, 21(5), 739-746.

25 D. A. Middleton, E. Hughes and M. Esmann, The Conformation of ATP within the Na,K-ATPase Nucleotide Site, Angew. Chem., 2011, 50(31), 7041-7044.

26 B. Y. Fang, M. H. Yao, C. Y. Wang, C. Y. Wang, Y. D. Zhao and F. Chen, Colloids Surf., B, 2016, 140, 233-238.

27 X. Liu, F. Wang, R. Aizen, O. Yehezkeli and I. Willner, J. Am. Chem. Soc., 2013, 135(32), 11832-11839.

28 C. Feng, S. Dai and L. Wang, Biosens. Bioelectron., 2014, 59(59C), 64-74.

29 K. Schiller, J. Heilmann, D. Manns and G. Franz, Planta Med., 2017, 4(S01), Mo-PO-187.

30 D. Haouzi, I. Cohen, H. L. A. Vieira, D. Poncet, P. Boya, M. Castedo and G. Feldmann, Apoptosis, 2002, 7(5), 395-405. 31 H. Jiang, L. Yang, X. Xing, M. Yan, X. Guo, B. Yang and H. X. Kuang, Phytomedicine, 2018, DOI: 10.1016/ j.phymed.2018.12.020.

32 F. Lu, M. Cao, B. Wu, X. Z. Li, H. Y. Liu, D. Z. Chen and S. M. Liu, J. Ethnopharmacol., 2013, 149(1), 311-320.

33 E. Pebay-Peyroula, C. Dahout-Gonzalez, R. Kahn, V. Trézéguet, G. J. M. Lauquin and G. Brandolin, Nature, 2003, 426(6962), 39-44.

34 A. Kedrov, A. M. Hellawell, A. Klosin, R. B. Broadhurst, E. R. Kunji and D. J. Müller, Structure, 2010, 18(1), 39. 